\title{
Explicit criteria for the qualitative properties of differential equations with $p$-Laplacian-like operator
}

\author{
Omar Bazighifan ${ }^{1,2^{*}}$ (D) and A.F. Aljohani ${ }^{3}$
}

\section{"Correspondence:}

o.bazighifan@gmail.com

'Department of Mathematics;

Faculty of Science, Hadhramout

University, Hadhramout 50512, Yemen

${ }^{2}$ Department of Mathematics; Faculty of Education, Seiyun University, Hadhramout 50512, Yemen

Full list of author information is available at the end of the article

\begin{abstract}
The aim of this work is to study qualitative properties of solutions for a fourth-order neutral nonlinear differential equation, driven by a $p$-Laplace differential operator. Some oscillation criteria for the equation under study have been obtained by comparison theory. The obtained results improve the well-known oscillation results present in the literature. Some examples are provided to show the applicability of the obtained results.
\end{abstract}

MSC: $34 \mathrm{C} 10 ; 34 \mathrm{~K} 11$

Keywords: Neutral differential equations; Oscillation; 4th order; $p$-Laplacian equation

\section{Introduction}

Differential equations of fourth-order appear in models concerning biological, physical, and chemical phenomena, optimization, mathematics of networks, dynamical systems, see [1].

We study the oscillatory behavior of the fourth-order neutral nonlinear differential equation of the form

$$
\left\{\begin{array}{l}
\left(r(x)\left|w^{\prime \prime \prime}(x)\right|^{p_{1}-2} w^{\prime \prime \prime}(x)\right)^{\prime}+\sum_{i=1}^{j} q_{i}(x)\left|u^{\prime \prime \prime}\left(\vartheta_{i}(x)\right)\right|^{p_{2}-2} u^{\prime \prime \prime}\left(\vartheta_{i}(x)\right)=0, \\
j \geq 1, \quad p_{2} \geq p_{1}, \quad r(x)>0, \quad r^{\prime}(x) \geq 0, \quad x \geq x_{0}>0,
\end{array}\right.
$$

where $w(x):=u(x)+a(x) u(\tau(x))$ and the first term means the $p$-Laplace-type operator $\left(1<p_{i}<\infty, i=1,2\right)$. The main results are obtained under the following conditions: $r \in C\left[x_{0}, \infty\right), a, q_{i} \in C\left[x_{0}, \infty\right), q_{i}(x)>0,0 \leq a(x)<a_{0}<1, \tau, \vartheta_{i} \in C\left[x_{0}, \infty\right), \tau(x) \leq x$, $\lim _{x \rightarrow \infty} \tau(x)=\lim _{x \rightarrow \infty} \vartheta_{i}(x)=\infty, i=1,2, \ldots, j$, and under the condition

$$
\int_{x_{0}}^{\infty} \frac{1}{r^{1 /\left(p_{1}-1\right)}(s)} \mathrm{d} s=\infty
$$

The $p$-Laplace equations have some significant applications in elasticity theory and continuum mechanics, see [2] (power-law fluids), and in general in nonlinear phenomena, see

(c) The Author(s) 2020. This article is licensed under a Creative Commons Attribution 4.0 International License, which permits use, sharing, adaptation, distribution and reproduction in any medium or format, as long as you give appropriate credit to the original author(s) and the source, provide a link to the Creative Commons licence, and indicate if changes were made. The images or other third party material in this article are included in the article's Creative Commons licence, unless indicated otherwise in a credit line to the material. If material is not included in the article's Creative Commons licence and your intended use is not permitted by statutory regulation or exceeds the permitted use, you will need to obtain permission directly from the copyright holder. To view a copy of this licence, visit http://creativecommons.org/licenses/by/4.0/. 
[3] (capillary phenomena). For some results concerning the oscillatory behavior of equations driven by a $p$-Laplace differential operator, we mention the papers [4-6].

In [7], the authors used a classical variational approach based on the critical points theory to prove the existence of at least one nontrivial weak solution of a double-phase Dirichlet problem. Here the differential operator of the problem is the sum of two $p$-Laplaciantype operators with variable exponents. This fact could provide new ideas for further investigations. The authors characterized the continuous spectrum of double-phase equations (to improve the regularity theory for such a kind of operators and classify solutions).

Nastasi [8] established an existence result of a nontrivial weak solution to $(p, q)$ - Laplacian problem on a noncompact Riemannian manifold. The special setting led the author to develop the Maz'ya's approach, by working with isocapacitary inequalities to characterize the compact embeddings.

\section{Mathematical background-hypotheses}

In this section we collect some relevant facts and auxiliary results from the existing literature. Also, we fix the notation.

Currently, researchers have become more concerned with the topic of oscillation of differential equations in [9-27]. Li et al. [4], using the Riccati transformation together with integral averaging technique, focused on the oscillations of the equation

$$
\left\{\begin{array}{l}
\left(r(x)\left|w^{\prime \prime \prime}(x)\right|^{p-2} w^{\prime \prime \prime}(x)\right)^{\prime}+\sum_{i=1}^{j} q_{i}(x)\left|y\left(\delta_{i}(x)\right)\right|^{p-2} y\left(\vartheta_{i}(x)\right)=0 \\
1<p<\infty, \quad x \geq x_{0}>0
\end{array}\right.
$$

In $[28,29]$, the comparison method with first and second order equations was used to investigate every solution $u$ of

$$
\left\{\begin{array}{l}
\left(r(x)\left|u^{(n-1)}(x)\right|^{p-2} u^{(n-1)}(x)\right)^{\prime}+\sum_{i=1}^{j} q_{i}(x) g\left(u\left(\vartheta_{i}(x)\right)\right)=0, \\
j \geq 1, \quad x \geq x_{0}>0,
\end{array}\right.
$$

where $n$ is even and $p>1$ is a real number, in the case where $\vartheta_{i}(x) \geq v$ (with $r \in$ $\left.C^{1}((0, \infty), \mathbb{R}), q_{i} \in C([0, \infty), \mathbb{R}), i=1,2, \ldots, j\right)$.

We point out that Bazighifan [30] gave us some results providing information on the oscillation of equations

$$
\left\{\begin{array}{l}
\left(r(x)\left|u^{\prime \prime \prime}(x)\right|^{p_{1}-2} u^{\prime \prime \prime}(x)\right)^{\prime}+q(x)\left|u^{\prime \prime \prime}\left(g_{1}(x)\right)\right|^{p_{2}-2} u^{\prime \prime \prime}\left(g_{1}(x)\right)=0, \\
r(x)>0, \quad r^{\prime}(x) \geq 0, \quad p_{2} \geq p_{1}, \quad x \geq x_{0}>0,
\end{array}\right.
$$

where $n$ is even. This time, the author used the comparison method with second order equations.

The authors of [31], using the Riccati technique, derived oscillation conditions of

$$
\left\{\begin{array}{l}
\left(r(x)\left|u^{(n-1)}(x)\right|^{p-2} u^{(n-1)}(x)\right)^{\prime}+q(x) g(u(\vartheta(x)))=0, \\
1<p<\infty
\end{array}\right.
$$

where $n$ is even. 
As we already mentioned in the Introduction, our aim here is to provide complementary results to $[28,29,31]$. For this purpose we briefly discuss these results.

Definition 2.1 Define sequences of functions $\left\{\delta_{n}(x)\right\}_{n=0}^{\infty}$ and $\left\{\sigma_{n}(x)\right\}_{n=0}^{\infty}$ as

$$
\begin{aligned}
& \delta_{0}(x)=\xi_{*}(x) \quad \text { and } \sigma_{0}(x)=\eta_{*}(x), \\
& \delta_{n}(x)=\delta_{0}(x)+\int_{x}^{\infty} R_{1}(x) \delta_{n-1}^{p_{1} /\left(p_{1}-1\right)}(s) \mathrm{d} s, \quad n \geq 1 \\
& \sigma_{n}(x)=\sigma_{0}(x)+\int_{x}^{\infty} \sigma_{n-1}^{p_{1} /\left(p_{1}-1\right)}(s) \mathrm{d} s, \quad n \geq 1 .
\end{aligned}
$$

We see by induction that $\delta_{n}(x) \leq \delta_{n+1}(x)$ and $\sigma_{n}(x) \leq \sigma_{n+1}(x)$ for $x \geq x_{0}, n \geq 1$.

Now, we are ready to introduce the precise hypotheses on the data of (1):

(H1) $u$ is an eventually positive solution of (1).

(H2) Let $B(x)=\left(p_{1}-1\right) \varepsilon \frac{\vartheta_{i}^{2}(x) \zeta \vartheta_{i}^{\prime}(x)}{r^{1 /\left(p_{1}-1\right)}(x)}$ and $\phi_{1}(x)=\int_{x}^{\infty} A(s) \mathrm{d} s$ be such that

$$
\liminf _{x \rightarrow \infty} \frac{1}{\phi_{1}(x)} \int_{x}^{\infty} B(s) \phi_{1}^{\frac{p_{1}}{\left(p_{1}-1\right)}}(s) \mathrm{d} s>\frac{p_{1}-1}{p_{1}^{p_{1} /\left(p_{1}-1\right)}},
$$

where

$$
A(x)=\sum_{i=1}^{j} q_{i}(x)\left(1-a_{0}\right)^{p_{2}-1} M^{p_{1}-p_{2}}\left(\vartheta_{i}(x)\right) .
$$

(H3) For some $\mu \in(0,1)$, there are positive constants $M_{1}, M_{2}$ such that

$$
\liminf _{x \rightarrow \infty} \frac{1}{\xi_{*}(x)} \int_{x}^{\infty} R_{1}(s) \xi_{*}^{p_{1} /\left(p_{1}-1\right)}(s) \mathrm{d} s>\frac{\left(p_{1}-1\right)}{p_{1}^{p_{1} /\left(p_{1}-1\right)}}
$$

and

$$
\liminf _{x \rightarrow \infty} \frac{1}{\eta_{*}(x)} \int_{x_{0}}^{\infty} \eta_{*}^{2}(s) \mathrm{d} s>\frac{1}{4}
$$

where

$$
\begin{aligned}
& R_{1}(x):=\left(p_{1}-1\right) \mu \frac{x^{2}}{2 r^{1 /\left(p_{1}-1\right)}(x)}, \\
& \xi(x):=\sum_{i=1}^{j} q_{i}(x)\left(1-a_{0}\right)^{p_{2}-1} M_{1}^{p_{2}-p_{1}} \varepsilon_{1}\left(\frac{\vartheta_{i}(x)}{x}\right)^{3\left(p_{2}-1\right)}, \\
& \eta(x):=\left(1-a_{0}\right)^{p_{2} / p_{1}} M_{2}^{p_{2} /\left(p_{1}-2\right)} \int_{x}^{\infty}\left(\frac{1}{r(\delta)} \int_{\delta}^{\infty} \sum_{i=1}^{j} q_{i}(s) \frac{\vartheta_{i}^{p_{2}-1}(s)}{s^{p_{2}-1}} \mathrm{~d} s\right)^{1 /\left(p_{1}-1\right)} \mathrm{d} \delta, \\
& \xi_{*}(x)=\int_{x}^{\infty} \xi(s) \mathrm{d} s \text { and } \eta_{*}(x)=\int_{x}^{\infty} \eta(s) \mathrm{d} s .
\end{aligned}
$$


(H4) For some $\mu_{1} \in(0,1)$, we have

$$
\limsup _{x \rightarrow \infty}\left(\frac{\mu_{1} x^{3}}{6 r^{1 /\left(p_{1}-1\right)}(x)}\right)^{p_{1}-1} \delta_{n}(x)>1
$$

and

$$
\limsup _{x \rightarrow \infty} \lambda x \sigma_{n}(x)>1
$$

for some $n$.

(H5) For some $n$, we have

$$
\int_{x_{0}}^{\infty} \xi(x) \exp \left(\int_{x_{0}}^{x} R_{1}(s) \delta_{n}^{1 /\left(p_{1}-1\right)}(s) \mathrm{d} s\right) \mathrm{d} x=\infty
$$

and

$$
\int_{x_{0}}^{\infty} \eta(x) \exp \left(\int_{x_{0}}^{x} \sigma_{n}^{1 /\left(p_{1}-1\right)}(s) \mathrm{d} s\right) \mathrm{d} x=\infty .
$$

\section{Main results}

Next, we mention some important lemmas:

Lemma 3.1 ([32]) Let $w$ satisfy $w^{(i)}(x)>0, i=0,1, \ldots, n$, and $w^{(n+1)}(x)<0$ eventually. Then, for every $\varepsilon_{1} \in(0,1), w(x) / w^{\prime}(x) \geq \varepsilon_{1} x / n$ eventually.

Lemma 3.2 ([10]) Let $w$ satisfy $w(x)>0$ and $w^{(n-1)}(x) w^{(n)}(x) \leq 0, x \geq x_{w}$, then there exist constants $\theta, 0<\theta<1$ and $\varepsilon>0$ such that

$$
w^{\prime}(\theta x) \geq \varepsilon x^{n-2} w^{(n-1)}(x)
$$

for all sufficiently large $x$.

Lemma $3.3([33])$ Let $w$ satisfy $w^{(n-1)}(x) w^{(n)}(x) \leq 0$ and $\lim _{x \rightarrow \infty} w(x) \neq 0$, then

$$
w(x) \geq \frac{\mu}{(n-1) !} x^{n-1}\left|w^{(n-1)}(x)\right| \quad \text { for } \mu \in(0,1) .
$$

Lemma 3.4 ([34]) If (H1) holds, then we can distinguish the following situations:

$\left(\mathbf{G}_{1}\right) \quad w^{(k)}(x)>0, \quad k=1,2,3$,

$\left(\mathbf{G}_{2}\right) \quad w^{(k)}(x)>0, \quad k=1,3$, and $w^{\prime \prime}(x)<0$,

for $x \geq x_{1}$, where $x_{1} \geq x_{0}$ is sufficiently large.

Theorem 3.1 If (H2) holds, then (1) is oscillatory. 
Proof Let (H1) hold, then there exists an $x_{1} \geq x_{0}$ such that $u(x)>0, u(\tau(x))>0$ and $u\left(\vartheta_{i}(x)\right)>0$ for $x \geq x_{1}$. Since $r^{\prime}(x)>0$, we have

$$
\begin{aligned}
& w(x)>0, \quad w^{\prime}(x)>0, \quad w^{\prime \prime \prime}(x)>0, \\
& w^{(4)}(x)<0 \quad \text { and } \quad\left(r(x)\left(w^{\prime \prime \prime}(x)\right)^{p_{1}-1}\right)^{\prime} \leq 0,
\end{aligned}
$$

for $x \geq x_{1}$. From the definition of $w$, we get

$$
u(x) \geq w(x)-a_{0} u(\tau(x)) \geq w(x)-a_{0} w(\tau(x)) \geq\left(1-a_{0}\right) w(x),
$$

which together with (1) gives

$$
\left(r(x)\left(w^{\prime \prime \prime}(x)\right)^{p_{1}-1}\right)^{\prime} \leq-\sum_{i=1}^{j} q_{i}(x)\left(1-a_{0}\right)^{p_{2}-1} w^{p_{2}-1}\left(\vartheta_{i}(x)\right) .
$$

Define

$$
\varpi(x):=\frac{r(x)\left(w^{\prime \prime \prime}(x)\right)^{p_{1}-1}}{w^{p_{1}-1}\left(\zeta \vartheta_{i}(x)\right)},
$$

for some a constant $\zeta \in(0,1)$. By differentiating the above and using (12), we get

$$
\begin{aligned}
\varpi^{\prime}(x) \leq & \frac{-\sum_{i=1}^{j} q_{i}(x)\left(1-a_{0}\right)^{p_{2}-1} w^{p_{2}-1}\left(\vartheta_{i}(x)\right)}{w^{p_{1}-1}\left(\zeta \vartheta_{i}(x)\right)} \\
& -\left(p_{1}-1\right) \frac{r(x)\left(w^{\prime \prime \prime}(x)\right)^{p_{1}-1} w^{\prime}\left(\zeta \vartheta_{i}(x)\right) \zeta \vartheta_{i}^{\prime}(x)}{w^{p_{1}}\left(\zeta \vartheta_{i}(x)\right)} .
\end{aligned}
$$

From Lemma 3.2, there exists a constant $\varepsilon>0$ such that

$$
\begin{aligned}
\varpi^{\prime}(x) \leq & -\sum_{i=1}^{j} q_{i}(x)\left(1-a_{0}\right)^{p_{2}-1} w^{p_{2}-p_{1}}\left(\vartheta_{i}(x)\right) \\
& -\left(p_{1}-1\right) \frac{r(x)\left(w^{\prime \prime \prime}(x)\right)^{p_{1}-1} \varepsilon \vartheta_{i}^{2}(x) w^{\prime \prime \prime}\left(\vartheta_{i}(x)\right) \zeta \vartheta_{i}^{\prime}(x)}{w^{p_{1}}\left(\zeta \vartheta_{i}(x)\right)},
\end{aligned}
$$

which implies

$$
\varpi^{\prime}(x) \leq-\sum_{i=1}^{j} q_{i}(x)\left(1-a_{0}\right)^{p_{2}-1} w^{p_{2}-p_{1}}\left(\vartheta_{i}(x)\right)-\left(p_{1}-1\right) \varepsilon \frac{r(x) \vartheta_{i}^{2}(x) \zeta \vartheta_{i}^{\prime}(x)\left(w^{\prime \prime \prime}(x)\right)^{p_{1}}}{w^{p_{1}}\left(\zeta \vartheta_{i}(x)\right)} .
$$

Using (13), we find

$$
\varpi^{\prime}(x) \leq-\sum_{i=1}^{j} q_{i}(x)\left(1-a_{0}\right)^{p_{2}-1} w^{p_{2}-p_{1}}\left(\vartheta_{i}(x)\right)-\left(p_{1}-1\right) \varepsilon \frac{\vartheta_{i}^{2}(x) \zeta \vartheta_{i}^{\prime}(x)}{r^{1 /\left(p_{1}-1\right)}(x)} \varpi^{p_{1} /\left(p_{1}-1\right)}(x)
$$

Since $w^{\prime}(x)>0$, there exist an $x_{2} \geq x_{1}$ and a constant $M>0$ such that

$$
w(x)>M .
$$


Then, (14) turns into

$$
\varpi^{\prime}(x) \leq-\sum_{i=1}^{j} q_{i}(x)\left(1-a_{0}\right)^{p_{2}-1} M^{p_{2}-p_{1}}\left(\vartheta_{i}(x)\right)-\left(p_{1}-1\right) \varepsilon \frac{\vartheta_{i}^{2}(x) \zeta \vartheta_{i}^{\prime}(x)}{r^{1 /\left(p_{1}-1\right)}(x)} \varpi^{p_{1} /\left(p_{1}-1\right)}(x)
$$

that is,

$$
\varpi^{\prime}(x)+A(x)+B(x) \varpi^{p_{1} /\left(p_{1}-1\right)}(x) \leq 0 .
$$

Integrating (15) from $x$ to $l$, we obtain

$$
\varpi(l)-\varpi(x)+\int_{x}^{l} A(s) \mathrm{d} s+\int_{x}^{l} B(s) \varpi^{p_{1} /\left(p_{1}-1\right)}(s) \mathrm{d} s \leq 0 .
$$

Letting $l \rightarrow \infty$ and using $\varpi>0$ and $\varpi^{\prime}<0$, we have

$$
\varpi(x) \geq \phi_{1}(x)+\int_{x}^{\infty} B(s) \varpi^{p_{1} /\left(p_{1}-1\right)}(s) \mathrm{d} s .
$$

This implies

$$
\frac{\varpi(x)}{\phi_{1}(x)} \geq 1+\frac{1}{\phi_{1}(x)} \int_{x}^{\infty} B(s) \phi_{1}^{p_{1} /\left(p_{1}-1\right)}(s)\left(\frac{\varpi(s)}{\phi_{1}(s)}\right)^{p_{1} /\left(p_{1}-1\right)} \mathrm{d} s .
$$

Let $\lambda=\inf _{x \geq x} \varpi(x) / \phi_{1}(x)$, then obviously $\lambda \geq 1$. So, from (4) and (16), we find

$$
\lambda \geq 1+\left(p_{1}-1\right)\left(\frac{\lambda}{p_{1}}\right)^{p_{1} /\left(p_{1}-1\right)}
$$

or

$$
\frac{\lambda}{p_{1}} \geq \frac{1}{p_{1}}+\frac{\left(p_{1}-1\right)}{p_{1}}\left(\frac{\lambda}{p_{1}}\right)^{p_{1} /\left(p_{1}-1\right)}
$$

which contradicts $\lambda \geq 1$ and $\left(p_{1}-1\right)>0$.

The proof is complete.

Theorem 3.2 If (H3) holds, then (1) is oscillatory.

Proof Let (1) have a nonoscillatory solution in $\left[x_{0}, \infty\right)$. Without loss of generality, we let $u(x)>0$. Then, there exists an $x_{1} \geq x_{0}$ such that $u(\tau(x))>0$ and $u\left(\vartheta_{i}(x)\right)>0$ for $x \geq x_{1}$. From Lemma 3.4, there are two cases $\left(\mathbf{G}_{1}\right)$ and $\left(\mathbf{G}_{2}\right)$.

For case $\left(\mathbf{G}_{1}\right)$, define

$$
\omega(x):=\frac{r(x)\left(w^{\prime \prime \prime}(x)\right)^{p_{1}-1}}{w^{p_{1}-1}(x)}
$$

From (12), we obtain

$$
\omega^{\prime}(x) \leq-\sum_{i=1}^{j} q_{i}(x)\left(1-a_{0}\right)^{p_{2}-1} \frac{w^{p_{2}-1}\left(\vartheta_{i}(x)\right)}{w^{p_{1}-1}(x)}-\left(p_{1}-1\right) \frac{r(x)\left(w^{\prime \prime \prime}(x)\right)^{p_{1}-1}}{w^{p_{1}}(x)} w^{\prime}(x) .
$$


From Lemma 3.1, we find

$$
\frac{w^{\prime}(x)}{w(x)} \leq \frac{3}{\varepsilon_{1} x}
$$

Integrating again from $\vartheta_{i}(x)$ to $x$, we find

$$
\frac{w\left(\vartheta_{i}(x)\right)}{w(x)} \geq \varepsilon_{1} \frac{\vartheta_{i}^{3}(x)}{x^{3}}
$$

It follows from Lemma 3.3 that

$$
w^{\prime}(x) \geq \frac{\mu_{1}}{2} x^{2} w^{\prime \prime \prime}(x)
$$

for all $\mu_{1} \in(0,1)$. Since $w^{\prime}(x)>0$, there exists an $x_{2} \geq x_{1}$ such that

$$
w(x)>M
$$

From (18), (19), (20), and (21), we obtain

$$
\omega^{\prime}(x)+\sum_{i=1}^{j} q_{i}(x)\left(1-a_{0}\right)^{p_{2}-1} M_{1}^{p_{2}-p_{1}} \varepsilon_{1}\left(\frac{\vartheta_{i}(x)}{x}\right)^{3\left(p_{2}-1\right)}+\frac{\left(p_{1}-1\right) \mu x^{2}}{2 r^{1 /\left(p_{1}-1\right)}(x)} \omega^{p_{1} /\left(p_{1}-1\right)}(x) \leq 0,
$$

that is,

$$
\omega^{\prime}(x)+\xi(x)+R_{1}(x) \omega^{p_{1} /\left(p_{1}-1\right)}(x) \leq 0 .
$$

Integrating (22) from $x$ to $l$, we find

$$
\omega(l)-\omega(x)+\int_{x}^{l} \xi(s) \mathrm{d} s+\int_{x}^{l} R_{1}(s) \omega^{p_{1} /\left(p_{1}-1\right)}(s) \mathrm{d} s \leq 0 .
$$

Letting $l \rightarrow \infty$ and using $\omega>0, \omega^{\prime}<0$, we get

$$
\omega(x) \geq \xi_{*}(x)+\int_{x}^{\infty} R_{1}(s) \omega^{p_{1} /\left(p_{1}-1\right)}(s) \mathrm{d} s .
$$

This implies

$$
\frac{\omega(x)}{\xi^{*}(x)} \geq 1+\frac{1}{\xi_{*}(x)} \int_{x}^{\infty} R_{1}(s) \xi_{*}^{p_{1} /\left(p_{1}-1\right)}(s)\left(\frac{\omega(s)}{\xi_{*}(s)}\right)^{p_{1} /\left(p_{1}-1\right)} \mathrm{d} s
$$

Let $\lambda=\inf _{x \geq x} \omega(x) / \xi_{*}(x)$, then $\lambda \geq 1$. So, from (5) and (24), we obtain

$$
\lambda \geq 1+\left(p_{1}-1\right)\left(\frac{\lambda}{p_{1}}\right)^{p_{1} /\left(p_{1}-1\right)},
$$

or

$$
\frac{\lambda}{p_{1}} \geq \frac{1}{p_{1}}+\frac{\left(p_{1}-1\right)}{p_{1}}\left(\frac{\lambda}{p_{1}}\right)^{p_{1} /\left(p_{1}-1\right)}
$$


which contradicts $\lambda \geq 1$ and $\left(p_{1}-1\right)>0$.

For case $\left(\mathbf{G}_{2}\right)$, integrating (12) from $x$ to $m$, we obtain

$$
r(m)\left(w^{\prime \prime \prime}(m)\right)^{p_{1}-1}-r(x)\left(w^{\prime \prime \prime}(x)\right)^{p_{1}-1} \leq-\int_{x}^{m} \sum_{i=1}^{j} q_{i}(s)\left(1-a_{0}\right)^{p_{2}-1} w^{p_{2}-1}\left(\vartheta_{i}(s)\right) \mathrm{d} s .
$$

From Lemma 3.1, we find

$$
w(x) \geq \varepsilon_{1} x w^{\prime}(x) \text { and hence } \quad w\left(\vartheta_{i}(x)\right) \geq \varepsilon_{1} \frac{\vartheta_{i}(x)}{x} w(x) .
$$

For (25), letting $m \rightarrow \infty$ and using (26), we see that

$$
r(x)\left(w^{\prime \prime \prime}(x)\right)^{p_{1}-1} \geq \varepsilon_{1}\left(1-a_{0}\right)^{p_{2}-1} w^{p_{2}-1}(x) \int_{x}^{\infty} \sum_{i=1}^{j} q_{i}(s) \frac{\vartheta_{i}^{p_{2}-1}(s)}{s^{p_{2}-1}} \mathrm{~d} s .
$$

Integrating (27) from $x$ to $\infty$, we obtain

$$
w^{\prime \prime}(x) \leq-\varepsilon_{1}\left(1-a_{0}\right)^{p_{2} / p_{1}} w^{p_{2} / p_{1}}(x) \int_{x}^{\infty}\left(\frac{1}{r(\delta)} \int_{\delta}^{\infty} \sum_{i=1}^{j} q_{i}(s) \frac{\vartheta_{i}^{p_{2}-1}(s)}{s^{p_{2}-1}} \mathrm{~d} s\right)^{1 /\left(p_{1}-1\right)} \mathrm{d} \delta,
$$

for all $\varepsilon_{1} \in(0,1)$. Define

$$
y(x)=\frac{w^{\prime}(x)}{w(x)} .
$$

By differentiating $y$ and from (21) and (28), we see that

$$
\begin{aligned}
y^{\prime}(x) \leq & -y^{2}(x) \\
& -\left(1-a_{0}\right)^{p_{2} / p_{1}} M^{\left(p_{2} / p_{1}\right)-1} \int_{x}^{\infty}\left(\frac{1}{r(\delta)} \int_{\delta}^{\infty} \sum_{i=1}^{j} q_{i}(s) \frac{\vartheta_{i}^{p_{2}-1}(s)}{s^{p_{2}-1}} \mathrm{~d} s\right)^{1 /\left(p_{1}-1\right)} \mathrm{d} \delta,
\end{aligned}
$$

hence

$$
y^{\prime}(x)+\eta(x)+y^{2}(x) \leq 0 .
$$

The rest of the proof of the case where $\left(\mathbf{G}_{2}\right)$ holds is the same as that of case $\left(\mathbf{G}_{1}\right)$. Thus, the proof is complete.

Theorem 3.3 If (H4) holds, then (1) is oscillatory.

Proof Proceeding as in the proof of Theorem 3.2, in the case $\left(\mathbf{G}_{1}\right)$, we see that (20) holds. By Lemma 3.3, we find

$$
w(x) \geq \frac{\mu_{1}}{6} x^{3} w^{\prime \prime \prime}(x) .
$$

From (17) and (31), we get

$$
\frac{1}{\omega(x)}=\frac{1}{r(x)}\left(\frac{w(x)}{w^{\prime \prime \prime}(x)}\right)^{p_{1}-1} \geq \frac{1}{r(x)}\left(\frac{\mu_{1}}{6} x^{3}\right)^{p_{1}-1} .
$$


Thus,

$$
\omega(x)\left(\frac{\mu_{1} x^{3}}{6 r^{1 /\left(p_{1}-1\right)}(x)}\right)^{p_{1}-1} \leq 1
$$

Therefore,

$$
\limsup _{x \rightarrow \infty} \omega(x)\left(\frac{\mu_{1} x^{3}}{6 r^{1 /\left(p_{1}-1\right)}(x)}\right)^{p_{1}-1} \leq 1
$$

which contradicts (7).

The rest of the proof is the same as that for the case $\left(\mathbf{G}_{2}\right)$. Theorem 3.3 is proved.

Corollary 3.1 If (H5) holds, then (1) is oscillatory.

Proof Proceeding as in the proof of Theorem 3.2, in the case $\left(\mathbf{G}_{1}\right)$, from (23) we obtain $\omega(x) \geq \delta_{0}(x)$.

By induction we can also see that $\omega(x) \geq \delta_{n}(x)$ for $x \geq x_{0}, n>1$. Since the sequence $\left\{\delta_{n}(x)\right\}_{n=0}^{\infty}$ is monotone increasing and bounded above, it converges to $\delta(x)$. Using Lebesgue's monotone convergence theorem, we find

$$
\delta(x)=\lim _{n \rightarrow \infty} \delta_{n}(x)=\int_{x}^{\infty} R_{1}(x) \delta^{p_{1} /\left(p_{1}-1\right)}(s) \mathrm{d} s+\delta_{0}(x)
$$

and

$$
\delta^{\prime}(x)=-R_{1}(x) \delta^{p_{1} /\left(p_{1}-1\right)}(x)-\xi(x) .
$$

Since $\delta_{n}(x) \leq \delta(x)$, it follows from (32) that

$$
\delta^{\prime}(x) \leq-R_{1}(x) \delta_{n}^{1 /\left(p_{1}-1\right)}(x) \delta(x)-\xi(x)
$$

Hence, we get

$$
\delta(x) \leq \exp \left(-\int_{x}^{x} R_{1}(s) \delta_{n}^{1 /\left(p_{1}-1\right)}(s) \mathrm{d} s\right)\left(\delta(x)-\int_{x}^{x} \xi(s) \exp \left(\int_{x}^{s} R_{1}(\delta) \delta_{n}^{1 /\left(p_{1}-1\right)}(\delta) \mathrm{d} \delta\right) \mathrm{d} s\right) .
$$

This implies

$$
\int_{x}^{x} \xi(s) \exp \left(\int_{x}^{s} R_{1}(\delta) \delta_{n}^{1 /\left(p_{1}-1\right)}(\delta) \mathrm{d} \delta\right) \mathrm{d} s \leq \delta(x)<\infty,
$$

which contradicts (9). The proof of the case where $\left(\mathbf{G}_{2}\right)$ holds is the same as that of $\left(\mathbf{G}_{1}\right)$. Corollary 3.1 is proved.

Example 3.1 Consider the differential equation

$$
\left(u(x)+\frac{1}{2} u\left(\frac{x}{2}\right)\right)^{(4)}+\frac{q_{0}}{x^{4}} u\left(\frac{x}{3}\right)=0
$$


where $q_{0}>0$. Let $p_{1}=p_{2}=2, r(x)=1, a(x)=1 / 2, \tau(x)=x / 2, \vartheta(x)=x / 3$, and $q(x)=q_{0} / x^{4}$. Then

$$
\begin{aligned}
& A(x)=\sum_{i=1}^{j} q_{i}(x)\left(1-a_{0}\right)^{\left(p_{2}-1\right)} M^{p_{2}-p_{1}}\left(\vartheta_{i}(x)\right)=\frac{q_{0}}{2 x^{4}} \\
& B(x)=\left(p_{1}-1\right) \varepsilon \frac{\vartheta_{i}^{2}(x) \zeta \vartheta_{i}^{\prime}(x)}{r^{1 /\left(p_{1}-1\right)}(x)}=\frac{\varepsilon x^{2}}{27}, \\
& \phi_{1}(x)=\frac{q_{0}}{6 x^{3}}
\end{aligned}
$$

and

$$
\begin{aligned}
& \liminf _{x \rightarrow \infty} \frac{1}{\phi_{1}(x)} \int_{x}^{\infty} B(s) \phi_{1}^{p_{1} /\left(p_{1}-1\right)}(s) \mathrm{d} s>\frac{\left(p_{1}-1\right)}{p_{1}^{p_{1} /\left(p_{1}-1\right)}}, \\
& \liminf _{x \rightarrow \infty} \frac{6 \varepsilon q_{0} x^{3}}{972} \int_{x}^{\infty} \frac{\mathrm{d} s}{s^{4}}>\frac{1}{4} \\
& q_{0}>121.5 \varepsilon
\end{aligned}
$$

for some $\varepsilon>0$. Thus, by Theorem 3.1, every solution of equation (33) is oscillatory if $q_{0}>$ $121.5 \varepsilon$.

Example 3.2 Consider a differential equation

$$
\left(u(x)+a_{0} u\left(\tau_{0} x\right)\right)^{(n)}+\frac{q_{0}}{x^{n}} u\left(\vartheta_{0} x\right)=0, \quad q_{0}>0 .
$$

Let $p=2, x_{0}=1, r(x)=1, a(x)=a_{0}, \tau(x)=\tau_{0} x, \vartheta(x)=\vartheta_{0} x$, and $q(x)=q_{0} / x^{n}$. Then we easily see that condition (5) holds and condition (6) is satisfied. Hence, by Theorem 3.2, every solution of equation (34) is oscillatory.

\section{Conclusions}

Our aim of this article was to study the qualitative behavior of a fourth-order neutral nonlinear differential equation, driven by a $p$-Laplace differential operator. The obtained oscillation theorems complement the well-known oscillation results present in the literature. In this line of work, one can investigate oscillatory conditions for a fourth-order equation of the type:

$$
\left\{\begin{array}{l}
\left(r(x)\left|y^{\prime \prime \prime}(x)\right|^{p_{1}-2} y^{\prime \prime \prime}(x)\right)^{\prime}+a(x) f\left(y^{\prime \prime \prime}(x)\right)+\sum_{i=1}^{j} q_{i}(x)\left|y\left(\sigma_{i}(x)\right)\right|^{p_{2}-2} y\left(\sigma_{i}(x)\right)=0 \\
x \geq x_{0}, \quad \sigma_{i}(x) \leq x, \quad j \geq 1, \quad 1<p_{2} \leq p_{1}<\infty .
\end{array}\right.
$$

which is of interest to the authors, in particular, the case of $p_{2}>p_{1}$.

\section{Acknowledgements}

The authors thank the editors and the reviewers for their useful comments.

Funding

The authors received no direct funding for this work.

Availability of data and materials

Not applicable. 


\section{Competing interests}

The authors declare that they have no competing interests.

\section{Authors' contributions}

The authors declare that the final manuscript has been read and approved.

\section{Author details}

${ }^{1}$ Department of Mathematics; Faculty of Science, Hadhramout University, Hadhramout 50512, Yemen. ${ }^{2}$ Department of Mathematics; Faculty of Education, Seiyun University, Hadhramout 50512, Yemen. ${ }^{3}$ Department of Mathematics, Faculty of Science, University of Tabuk, Tabuk, Saudi Arabia.

\section{Publisher's Note}

Springer Nature remains neutral with regard to jurisdictional claims in published maps and institutional affiliations.

Received: 14 July 2020 Accepted: 17 August 2020 Published online: 01 September 2020

\section{References}

1. Hale, J.K.: Theory of Functional Differential Equations. Springer, New York (1977)

2. Aronsson, G., Janfalk, U.: On Hele-Shaw flow of power-law fluids. Eur. J. Appl. Math. 3, 343-366 (1992)

3. Vetro, C.: Pairs of nontrivial smooth solutions for nonlinear Neumann problems. Appl. Math. Lett. 103, 106171, 1-7 (2020)

4. Li, T., Baculikova, B., Dzurina, J., Zhang, C.: Oscillation of fourth order neutral differential equations with $p$-Laplacian like operators. Bound. Value Probl. 2014, 56 (2014)

5. Liu, S., Zhang, Q., Yu, Y.: Oscillation of even-order half-linear functional differential equations with damping. Comput. Math. Appl. 61, 2191-2196 (2011)

6. Zhang, C., Agarwal, R., Li, T.: Oscillation and asymptotic behavior of higher-order delay differential equations with p-Laplacian like operators. J. Math. Anal. Appl. 409, 1093-1106 (2014)

7. Vetro, C., Vetro, F.: On problems driven by the $(p(\cdot), q(\cdot))$-Laplace operator. Mediterr. J. Math. 17(24), 1-11 (2020)

8. Nastasi, A.: Weak solution for Neumann $(p, q)$-Laplacian problem on Riemannian manifold. J. Math. Anal. Appl. 479, 45-61 (2019)

9. Baculikova, B., Dzurina, J.: Oscillation theorems for second-order nonlinear neutral differential equations. Comput. Math. Appl. 62, 4472-4478 (2011)

10. Bazighifan, O:: Kamenev and Philos-type oscillation criteria for fourth-order neutral differential equations. Adv. Differ Equ. 2020, 201, 1-12 (2020)

11. Agarwal, R., Grace, S.R., O'Regan, D.: Oscillation criteria for certain $n$th order differential equations with deviating arguments. J. Math. Anal. Appl. 262, 601-622 (2001)

12. Baculikova, B., Dzurina, J.: On the oscillation of odd order advanced differential equations. Bound. Value Probl. 2014, 214 (2014)

13. Bazighifan, O., Elabbasy, E.M., Moaaz, O.: Oscillation of higher-order differential equations with distributed delay. J. Inequal. Appl. 2019, 55, 1-9 (2019)

14. Bazighifan, O., Ramos, H.: On the asymptotic and oscillatory behavior of the solutions of a class of higher-order differential equations with middle term. Appl. Math. Lett. 107, 106431 (2020)

15. Li, T., Baculikova, B., Dzurina, J: Oscillatory behavior of second-order nonlinear neutral differential equations with distributed deviating arguments. Bound. Value Probl. 2014, 68 (2014)

16. Grace, S., Agarwal, R., Graef, J.: Oscillation theorems for fourth order functional differential equations. J. Appl. Math. Comput. 30, 75-88 (2009)

17. Gyori, I., Ladas, G.: Oscillation Theory of Delay Differential Equations with Applications. Clarendon, Oxford (1991)

18. Moaaz, O., Dassios, I., Bazighifan, O., Muhib, A.: Oscillation theorems for nonlinear differential. Equations of fourth-order. Mathematics 8, 520 (2020)

19. Ragusa, M.A.: Homogeneous Herz spaces and regularity results. Nonlinear Anal., Theory Methods Appl. 71, e1909-e1914 (2009)

20. Philos, Ch.G.: On the existence of non-oscillatory solutions tending to zero at $\infty$ for differential equations with positive delays. Arch. Math. 36, 168-178 (1981)

21. Agarwal, R.P., Gala, S., Ragusa, M.A.: A regularity criterion in weak spaces to Boussinesq equations. Mathematics 8(6), 920 (2020)

22. Ragusa, M.A., Tachikawa, A.: Regularity for minimizers for functionals of double phase with variable exponents. Adv. Nonlinear Anal. 9, 710-728 (2020)

23. Bazighifan, O., Cesarano, C.: Some new oscillation criteria for second order neutral differential equations with delayed arguments. Mathematics 7, 1-9 (2019)

24. Cesarano, C., Bazighifan, O.: Oscillation of fourth-order functional differential equations with distributed delay. Axioms $8,1-7(2019)$

25. Elabbasy, E.M., Cesarano, C., Bazighifan, O., Moaaz, O.: Asymptotic and oscillatory behavior of solutions of a class of higher order differential equation. Symmetry 11, 1-9 (2019)

26. Cesarano, C., Pinelas, S., Al-Showaikh, F., Bazighifan, O.: Asymptotic properties of solutions of fourth-order delay differential equations. Symmetry 11, 1-10 (2019)

27. Moaaz, O., Cesarano, C., Muhib, A.: Some new oscillation results for fourth-order neutral differential equations. Eur. J. Pure Appl. Math. 13, 185-199 (2020)

28. Bazighifan, O., Kumam, P.: Oscillation theorems for advanced differential equations with p-Laplacian like operators. Mathematics 8, 821 (2020)

29. Bazighifan, O., Abdeljawad, T.: Improved approach for studying oscillatory properties of fourth-order advanced differential equations with p-Laplacian like operator. Mathematics 8, 656 (2020) 
30. Bazighifan, O.: On the oscillation of certain fourth-order differential equations with p-Laplacian like operator. Appl. Math. Comput. 386, 125475 (2020)

31. Park, C., Moaaz, O., Bazighifan, O.: Oscillation results for higher order differential equations. Axioms 9, 1-10 (2020)

32. Chatzarakis, G.E., Grace, S.R., Jadlovska, I., Li, T., Tunc, E.: Oscillation criteria for third-order Emden-Fowler differential equations with unbounded neutral coefficients. Complexity 2019, 1-7 (2019)

33. Agarwal, R., Grace, S., O'Regan, D.: Oscillation Theory for Difference and Functional Differential Equations. Kluwer Academic, Dordrecht (2000)

34. Chatzarakis, G.E., Elabbasy, E.M., Bazighifan, O.: An oscillation criterion in 4th-order neutral differential equations with a continuously distributed delay. Adv. Differ. Equ. 2019, 336, 1-9 (2019)

Submit your manuscript to a SpringerOpen ${ }^{\circ}$ journal and benefit from:

- Convenient online submission

- Rigorous peer review

- Open access: articles freely available online

- High visibility within the field

- Retaining the copyright to your article

Submit your next manuscript at $\gg$ springeropen.com 